\title{
Discriminating multiple JPEG compressions using first digit features
}

\author{
SIMONE MILANI, MARCO TAGLIASACCHI AND STEFANO TUBARO
}

\begin{abstract}
The analysis of JPEG double-compressed images is a problem largely studied by the multimedia forensics community, as it might be exploited, e.g., for tampering localization or source device identification. In many practical scenarios, like photos uploaded on blogs, on-line albums, and photo sharing web sites, images might be JPEG compressed several times. However, the identification of the number of compression stages applied to an image remains an open issue. We proposes a forensic method based on the analysis of the distribution of the first significant digits of the discrete cosine transform coefficients, which follow Benford's law in images compressed just once. Then, the detector is optimized and extended in order to identify accurately the number of compression stages applied to an image. The experimental validation considers up to four consecutive compression stages and shows that the proposed approach extends and outperforms the previously-published algorithms for double JPEG compression detection.
\end{abstract}

Keywords: Multiple JPEG compression, Forgery identification, First digit features, Benford's law.

Received 30 June 2014; Revised 5 December 2014; Accepted 5 December 2014

\section{INTRDDUCTIDN}

The recent development of multimedia devices and editing tools, together with the proliferation of communication infrastructures and content sharing applications, has made the acquisition, the editing, and the diffusion of images and videos relatively-easy tasks. A single multimedia content downloaded from the internet can be the outcome of a long chain of processing steps. This fact introduces several concerns about the origin, the authenticity, and the trustability of images and videos downloaded from the network $[1,2]$. Moreover, identifying the origin and the authenticity (i.e., the absence of alterations after acquisition) of images proves to be a crucial element in court cases for the validation of evidences [3]. Moreover, detecting alterations permits inferring an objective quality evaluation of the analyzed multimedia content.

From these premises, multimedia forensic analysts have been recently focusing on detecting alterations on images since they can be easily acquired and modified even by a non-expert user. In order to fulfill this task, many of the proposed works aim at identifying images, which have been compressed more than once estimating the coding parameters that characterize the coding stages that precede the last one [4]. This fact is justified by the observation that most

Dipartimento di Elettronica, Informazione, e Bioingegneria (DEIB), Politecnico di Milano, P.za Leonardo da Vinci 32, 20133 Milano, Italy

Corresponding author:

S. Milani

Email: simone.milani@polimi.it of the digital multimedia contents are available in compressed format. Indeed, most of the images distributed over the internet are coded according to the JPEG standard [5].

All the solutions proposed in the literature aim at detecting double compression on images assuming that some alterations can be performed between the first and the second compression stages. However, we believe that this assumption does not hold in many practical scenarios since analyzing the feasible processing chains for a given downloaded image it is deducible that more than two compression stages may have been applied. As an illustrative example, let us consider an image, which is originally compressed by the acquisition device (i.e., a video or photo camera) to be stored in the onboard memory. A second compression is performed by the owner, after editing the image to enhance the perceptual quality and adjust the format (e.g., brightness/contrast adjustment, rescaling, cropping, color correction, etc.). A third compression is performed whenever the content is uploaded to a blog or to an on-line photo album. As a matter of fact, it is reasonable to assume that a large number of digital images available online have gone through more than two compression stages performed by its owner, and could be further compressed by other users. In these cases, a method that identifies the number of compression stages proves to be extremely important in reconstructing the processing history of a content [6].

This paper aims at identifying traces of multiple JPEG compression reconstructing the number of compression stages that have been operated on it. The proposed approach extends some of the techniques adopted by previous double 
compression detectors, which typically look for irregularities in the statistics of discrete cosine transform (DCT) coefficients [7, 8]. More precisely, our analysis considers the most significant decimal digit or first digit (FD) of DCT coefficient absolute values. For images compressed only once, the probability mass function (pmf) of FDs presents a regular behavior modelled by the so-called Benford's law [9]. Conversely, the pmf of FDs for double or multiple compressed images departs from this behavior [10].

Even if several literature contributions have analyzed this fact (e.g., [4]), we provide some experimental results that clearly show the validity of this assertion. Moreover, in the simplified hypothesis that DCT coefficients follows a Laplacian statistics [11].

Then, it is showed that the characteristics of these deviations can be related to the number of compressions applied to the content. Experimental results show that it is indeed possible to infer the number of compression stages from the FD statistics with an average accuracy around $85 \%$.

The proposed technique can be employed in different application scenarios, including steganalysis [7], detection of image manipulation (when the original image is decompressed, modified, and recompressed), forgery identification [12], and quality assessment. As an example, image tampering can be localized by looking for regions that have been compressed a different amount of times with respect to the remaining image. Moreover, given the correlation between the compression operations and the editing stages applied on an image, the authenticity and the real origin of an image can be discussed by analyzing the number of compressions.

The rest of the paper is organized as follows. Section II overviews some of the published works related to double compression. Section III describes the behavior of FD statistics, as they go through multiple quantization stages, while Section IV presents the proposed classification method. Experimental results are reported in Section V and final conclusions are drawn in Section VI.

\section{RELATED WORKS}

Although the problem of multiple compression detection is relatively new in the multimedia forensics, several works on double compression detection have been presented in the literature. Some of the proposed solutions analyze the statistics of DCT coefficients [7]. The second compression implies a re-quantization of DCT coefficients that are redistributed among the bins of the second quantizer following a different statistics with respect to single compressed images. In [13], Lin et al. observe that double quantization introduces periodicities in coefficient statistics, which can be exploited to reveal double compression and the set of parameters used. Similarly, the work in [7] detects the peculiar modifications on coefficients statistics brought by double quantization using an support vector machine (SVM) classifier. The approach in [14] generalizes the Benford's law for the DCT coefficients and checks its consistency on the analyzed image (more details will be provided in Section III). Other strategies rely on the assumption that quantization is an idempotent operation, i.e., requantizing DCT coefficients with the same quantizer leads to reconstructed values highly-correlated with its input [15]. In [16], the authors detect double compression by studying coding artifacts. In [17] a convolutive mixing model is presented in order to deal with the problem of shifting the image between the first and the second compression. Other methods rely on modelling the statistics of natural images. These include the approaches based on the analysis of the distribution of the first significant digits, which can be modeled according to Benford's law [18].

Many of these solutions are employed to detect tampering [4], steganalysis [7], or forgery localization [19]. The main idea lying at the base of these approaches is that distinguishing double compressed regions from single compressed regions permit identifying which parts have been modified and which are original.

In the following, we will show how it is possible to extend the approaches based on Benford's law to the detection of multiple compression on images.

\section{MULTIPLE COMPRESSIONS AND CDEFFICIENTS STATISTICS}

\section{A) The JPEG compression standard}

The JPEG image compression standard defines a blockbased transform coder (see Fig. 1), which partitions the input image into $8 \times 8$ pixel blocks $\mathbf{X}$ and computes the DCT of each block. Transform coefficients $\mathbf{Y}$ are quantized into integer-valued quantization levels $\mathbf{Y}_{\Delta_{1}}^{1}$

$$
Y_{\Delta_{1}}^{1}(i, j)=\operatorname{sign}(Y(i, j)) \text { round }\left(\frac{|Y(i, j)|}{\Delta_{1}(i, j)}\right),
$$

where the indexes $(i, j), i, j=0, \ldots, 7$, denote the position of the elements in the $8 \times 8$ block. The values $Y_{\Delta_{1}}^{1}(i, j)$ are converted into a binary stream by an entropy coder following a zig-zag scan that orders coefficients according to increasing spatial frequencies. The coded block can be reconstructed by applying an inverse DCT transform on the rescaled coefficients $Y_{r}^{1}(i, j)=Y_{\Delta_{1}}^{1}(i, j) \cdot \Delta_{1}(i, j)$. Note that the quantization step $\Delta_{1}(i, j)$ changes according to the index $(i, j)$ of the DCT coefficient and is usually

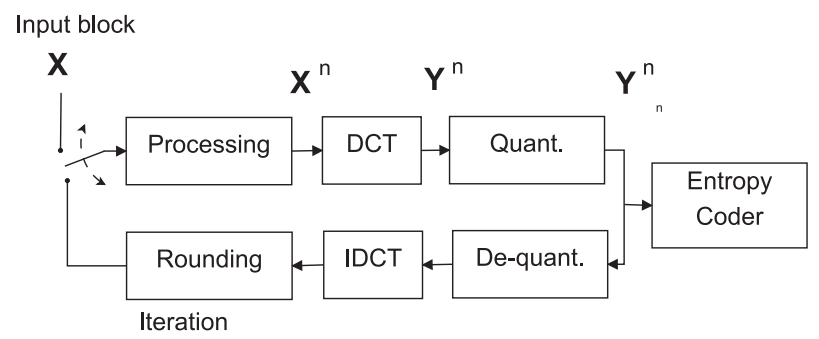

Fig. 1. Bock diagram for multiple JPEG compression. 
defined by means of a quantization matrix. In the Independent JPEG Group (IJG) implementation, the quantization matrix is selected by adjusting a quality factor $(\mathrm{QF})$, which varies in the range $[0,100]$. The higher $Q F$, the higher the quality of the constructed image.

When the image is encoded a second time, the resulting quantization levels are

$Y_{\Delta_{2}}^{2}(i, j)=\operatorname{sign}\left(Y_{\Delta_{1}}^{1}(i, j)\right)$ round $\left(\frac{\left|Y_{\Delta_{1}}^{1}(i, j) \cdot \Delta_{1}(i, j)\right|}{\Delta_{2}(i, j)}\right)$,

where $\Delta_{2}(i, j)$ are the quantization steps of the second compression stage. ${ }^{1}$ It is possible to iterate the compression process $N$ times leading to the quantization levels $Y_{\Delta_{N}}^{N}(i, j)$.

\section{B) The FD law}

Many fraud detection algorithms departs from the assumption that the analyzed data are well modeled by pre-defined stochastic models that present peculiar characteristics. A similar assumption is adopted by many approaches aiming at detecting double JPEG compression. More precisely, some of the proposed works rely on detecting the violation of the so-called Benford's law (also known as FD law or significant digit law) [20]. Many fraud detection algorithms departs from the assumption that the analyzed data are well modeled by pre-defined stochastic models that present peculiar characteristics. One of these is the FD rule, which proves to be satisfied for many distributions. The most significant digit or FD for a strictly-positive integer $Y$ (in base-10 notation) can be computed as

$$
m=\mathrm{FD}(Y)=\left\lfloor\frac{Y}{\left.10^{\left\lfloor\log _{10} Y\right\rfloor}\right\rfloor .}\right.
$$

It is possible to state that Benford's law [9] is satisfied whenever the pmf of $m$ can be well approximated by the equation

$$
\begin{array}{lr}
p(m)=N \log _{10}\left(1+\frac{1}{m}\right) & \text { or } \\
p(m)=N \log _{10}\left(1+\frac{1}{\beta+m^{\alpha}}\right) & \text { (generalized), }
\end{array}
$$

where $N$ is a normalizing factor and $\alpha, \beta$ are the parameters characterizing the model. This property can be verified for many real-life sources of data and can be effectively used to detect alterations on the analyzed data. Indeed, whenever some kind of modification has been performed on a dataset that originally satisfies Benford's law, the resulting distribution no longer satisfies this property. This fact has been widely employed in fraud detection in different fields (elections $[21,22]$, budget $[23,24]$, etc.), as well as in detecting double compression in JPEG images [14].

${ }^{1}$ Note that in this model we omit to consider the rounding and clipping of the reconstructed pixels to finite precision integer values after inverse DCT. This approximation is allowed by the fact that the effects of quantization prevail over those of rounding.

\section{C) Statistics of coefficients and FDs after multiple compressions}

The single and double compressions of an image leave different peculiar traces on the statistics of coefficients and their FDs. Most of the double compression detectors use these traces to decide whether an image has been compressed once or not. For the sake of conciseness, we will refer to the absolute DCT quantized coefficients after the $N$ th compression as $y_{N}=\left|Y_{\Delta_{N}}^{N}\right|$ (position indexes $(i, j)$ have been omitted for the sake of clarity) and their corresponding FDs $m_{N}=\operatorname{FD}\left(y_{N}\right)$.

When multiple compressions are applied, each compression stage modifies the statistics of coefficients and their corresponding FDs leaving denotative elements.

Fig. 2 reports the pmf of FDs for DCT coefficients at different coding stages for the image house of the Kodak dataset. The adopted quantization factors are $Q F_{4}=85$, $Q F_{3}=76, Q F_{2}=80$, and $Q F_{1}=85$. The same figure also reports the discrete fourier transform (DFT) of the difference (parameter $\chi$ ) between the actual pmf of FDs and its interpolated version following Benford's law, i.e., $\chi\left(m_{N}\right)=P\left[m_{N}=k\right]-p\left(m_{N}\right)$. Figures $2(\mathrm{e})-2(\mathrm{~h})$ show that the most significant oscillations take place at peculiar normalized frequencies of DFT coefficients. For single compressed images, oscillations are quite limited (DFT coefficients are lower than 0.17). In case an image is double compressed (Fig. 2(f)), part of the energy of $\chi$ is concentrated around $F=0.2$ (where $F$ is the normalized frequency for DFT transform). Note that in this case the maximum value of DFT coefficients is higher with respect to the case of images compressed once. For images compressed three times, the DFT plots show that two oscillating modes appear at $F \simeq 0.18$ and $F \simeq 0.28$ (Fig. 2 (g)), while four compressions lead to stronger oscillations dominated by the component at $F=0.34$ (FFT results highly peaked in Fig. 2(h)).

Ad-hoc solutions need to be designed in order to reveal the presence of these oscillations in the statistics of the FD for transform coefficients. In fact, previous solutions targeted simple double compression detection, i.e., were aimed at verifying the regularity of the actual FD statistics $\hat{p}(m)$. Multiple compression detection requires characterizing the characteristics of deviations from Benford's law. As a result, double compression detectors fail in discriminating the number of coding stages and the corresponding coding parameters.

It is possible to overcome this problem by combining a set of classifiers, as it will be described in the next section.

\section{THE PRDPDSED DETECTION ALGDRITHM}

The estimation of the number of coding stages $N$ requires to select a set of robust features that present a strong correlation with the traces left by quantization. From this selection, it is possible to design a set of classifiers associated 
(a)

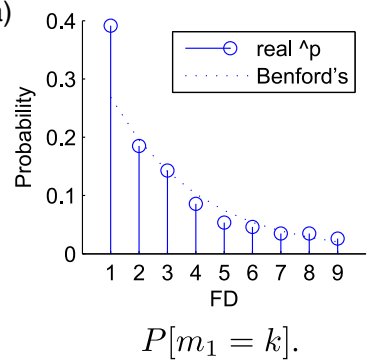

(e)

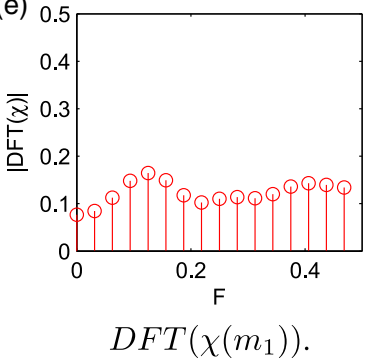

(b)

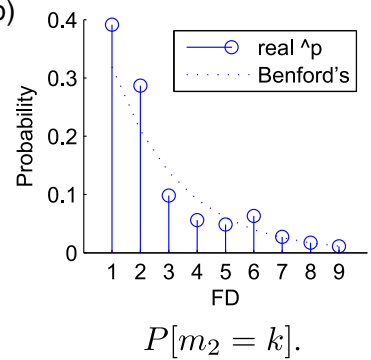

(f)

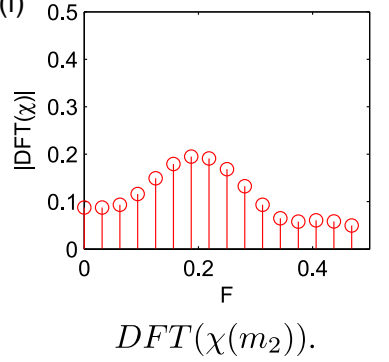

(c)

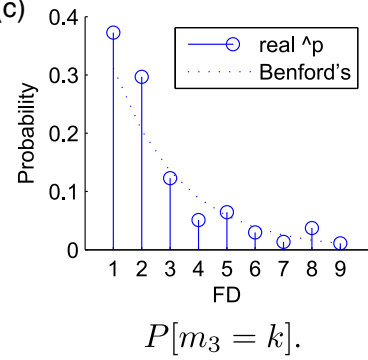

(g)

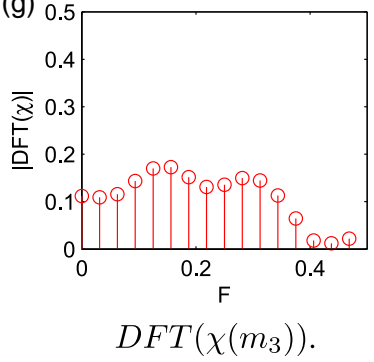

(d) 0.4

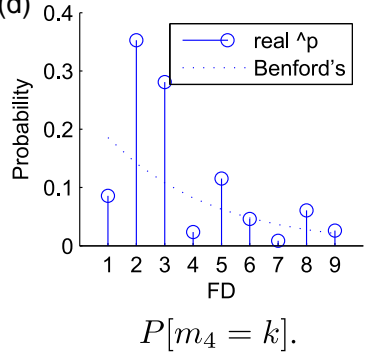

(h)

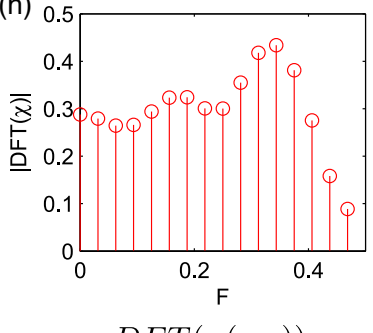

Fig. 2. Probability mass functions of FDs and frequency spectra of their differences $\chi\left(y_{N}\right)$ with respect to a Benford's model at different coding stages. The parameter $F$ denotes the normalized frequency for DFT coefficients of $\chi$. The graphs are related to coefficient at position $(1,0)$ for image house. (a) $P\left[m_{1}=k\right]$. (b) $P\left[m_{2}=k\right]$. (c) $P\left[m_{3}=k\right]$. (d) $P\left[m_{4}=k\right]$. (e) $D F T\left(\chi\left(m_{1}\right)\right)$. (f) $D F T\left(\chi\left(m_{2}\right)\right)$. (g) $D F T\left(\chi\left(m_{3}\right)\right)$. (h) $D F T\left(\chi\left(m_{4}\right)\right)$.

to each compression stage. Their outputs can be combined estimating the number of compressions operated on the image under analysis.

To this purpose, we generated an exhaustive dataset of compressed images, where the number of compressions operated on each picture can vary from 1 to 4 . The adopted JPEG codec is the one implemented in the MATLAB software. The quality factor $Q F_{i}$ of the $i$ th compression stage is selected randomly within an interval of possible values $\left[Q F_{N}-d Q F, Q F_{N}+d Q F\right]$, where $Q F_{N}$ is the QF of the last compression stage and $d Q F$ is the halved width of the interval. This limit was imposed in order to constrain the quality degradation in the final image since significant variations in the QF values across the different compression stages introduce evident unnatural artifacts on the reconstructed pictures.

Moreover, we also imposed that the difference between the chosen QFs of two consecutive compression stages must be higher than a threshold $T_{Q F}$, i.e. $\left|Q F_{i}-Q F_{i+1}\right|>T_{Q F}$. This constraint was introduced in order to make the quantization steps for all the considered DCT coefficients change from one compression stage to the following.

In the literature, the set and the number of analyzed DCT coefficients employed by different double compression detectors can change. In [18], 20 spatial frequencies were considered, while in the approach [7] only 9 frequencies are considered. Note that most of these frequencies lies at low frequencies since DCT coefficients at low frequencies proves to be more regular and changes less significantly according to the characteristics of the image with respect to those located at high frequencies. Moreover, high-frequency coefficients are often quantized to zero, and therefore, their statistics can only be computed from a limited amount of data. In this approach, we adopted the set of coefficients in [7]. Then, we set $T_{Q F}=5$ since, according to the values of the quantization matrix of the JPEG codec implemented by MATLAB, it ensures that none of the DCT coefficients at compression stage $i$ is quantized with $\Delta_{i}=$ $\Delta_{i-1}$.

Three different datasets were generated. In a first dataset $D_{0}$, the compression grids of images are aligned, i.e., no transformation was operated on the image between one compression stage and the following. In a second dataset $D_{1}$, images were rescaled at a random compression stage, where the rescaling factor was randomly-chosen in the interval $[0.9,1.1]$. In the third dataset $D_{2}$, images are rotated of a random angle included in the interval $[-5,5]$ at a randomly-selected compression stage. In this way, datasets $D_{1}$ and $D_{2}$ introduce the possibility that the compression grids could not be aligned (since they include both transformed and unaltered images).

In the following, we will describe the feature extraction process and the training of the classifiers for the dataset $D_{0}$. The same operations are applied on the datasets $D_{1}$ and $D_{2}$ as well.

\section{A) Feature selection}

Given a JPEG image $I^{n}$ which has been compressed $n$ times, most of the approaches existing in the literature extract the quantized DCT coefficients of the luma component and compute a set of features from their statistics. Features may include the simple FD statistics for the DCT coefficients at low frequencies [18] or the relative difference between the actual pmf and the Benford's equation, i.e. $\chi(m)=$ $(p(m)-\hat{p}(m)) / \hat{p}(m)[14]$.

In our approach, we adopted the first possibility computing the pmfs of FDs on the set of nine spatial frequencies reported in [7]. This would lead to a feature array of 

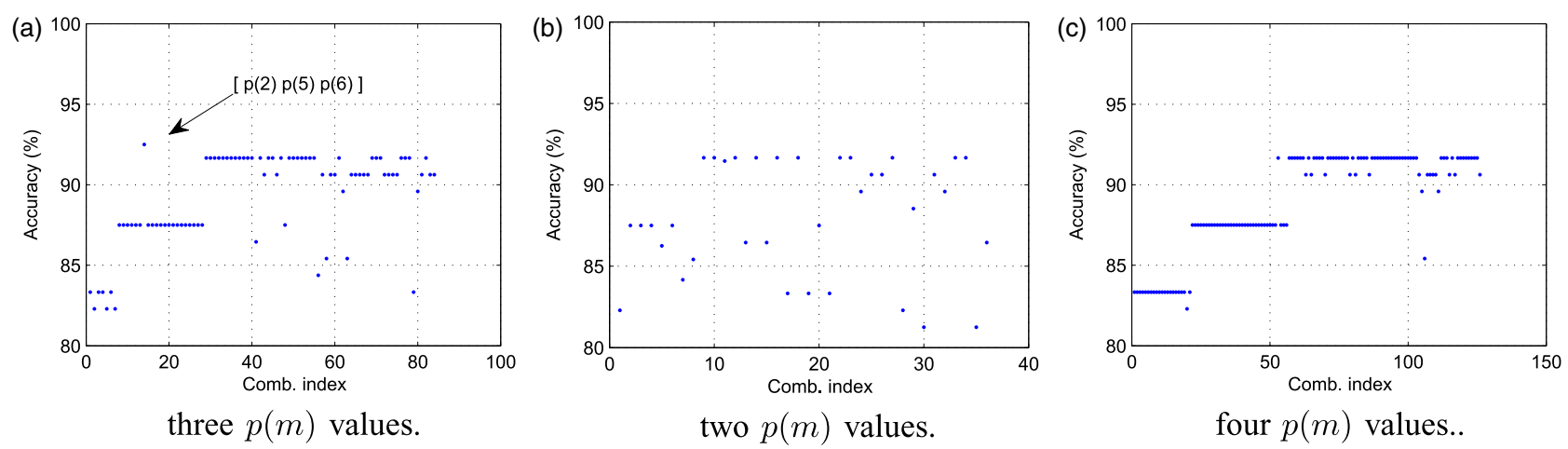

Fig. 3. Accuracy of single versus double compression detector for different feature arrays. (a) three $p(m)$ values, (b) two $p(m)$ values, and (c) four $p(m)$ values.

$9 \times 8=72$ values, ${ }^{2}$ but we aim at reducing this size by properly selecting those features that are extremely sensible to the number of compression stages. More precisely, Fig. 2 shows that the probabilities for some FD values are more sensitive to multiple compression than others, i.e., the deviation from Benford's law equation is more discriminative. From this premise, it is possible to select a subset of $l$ probability values $p\left(k_{1}\right), \ldots, p\left(k_{l}\right)$ from the pmf of FD digits $\left(k_{1}, \ldots, k_{2} \in[1,9]\right)$.

Naming $\hat{p}_{F_{h}, m}(k)=P_{F_{h}}[m==k]$ the probability that the FD value $m$ for the coefficients located at spatial frequency $F_{h}$ is equal to $k$, it is possible to gather the features in a common array

$$
\begin{aligned}
\mathbf{f}= & {\left[p_{F_{1}, m}\left(k_{1}\right) \cdots p_{F_{1}, m}\left(k_{l}\right) p_{F_{2}, m}\left(k_{1}\right) \cdots p_{F_{2}, m}\left(k_{l}\right) \cdots\right.} \\
& \left.\times p_{F_{9}, m}\left(k_{1}\right) \cdots p_{F_{9}, m}\left(k_{l}\right)\right] .
\end{aligned}
$$

Note that features are already limited in the range $[0,1]$ since they are related to pmf of FD values.

In order to find the optimal set of features, we considered different sets of $\hat{p}(m)$ values changing both the values $k_{1}, \ldots, k_{l}$ and their number $l$. To this purpose, we randomly selected 1200 images from the UCID dataset [25] which were compressed once and twice with different quantization parameters $Q F$. The quantization parameter of the last coding stage is fixed (since it is known from the coded bit stream), while the $Q F$ adopted in the first stage is randomly chosen in the interval $[Q F-10, Q F-5] \cup$ $[Q F+5, Q F+10]$. In this part, 10 different realizations were generated for every image. From these images, we computed the vectors $\mathbf{f}$ and used them in training a binary SVM classifier that discriminates single from double compressed images. The remaining 100 images in the UCID dataset were double compressed in a similar manner and used for testing the accuracy of the classifier. In this case, the double compression detector is taken as a simplification of the multiple compression detector for the sake of complexity since training the full multiple compression detector for all the configurations would require a significant time.

${ }^{2}$ In this case, one of the nine probability values of FD statistics can be omitted since it linearly dependent on the other values.
Fig. 3(a) reports the average detection using a feature array $\mathbf{f}$ with $l=3$, where the feature array has been created selecting only a subset $\left(k_{1}, k_{2}, k_{3}\right)$ of possible FD values, i.e., $\mathbf{f}=\left[p_{F_{h}, m}\left(k_{1}\right) p_{F_{h}, m}\left(k_{2}\right) p_{F_{h}, m}\left(k_{3}\right)\right]_{F_{h}}$. The different results have been obtained considering all the possible combinations for $k_{1}, k_{2}, k_{3}$. For the sake of clarity, the triplet $\left[k_{1}, k_{2}, k_{3}\right]$ is indexed by the value $\left(k_{1}-1\right)+\left(k_{2}-1\right) 9+$ $\left(k_{3}-1\right) 81$ (reported on the $x$-axis). It is possible to see that the triplet $[2,5,6]$ permits obtaining the highest accuracy. Note also that the length of the feature vector is 27 . It is also possible to verify that the obtained accuracy is comparable with that obtained by decomposing the feature array f with $l=8$ using a principal component analysis (PCA) and selecting the three most relevant components. Building a new classifier on this feature array obtained from PCA, it is possible to obtain an average accuracy of $92 \%$. Nevertheless, the PCA-based classifier requires computing all the features in advance and then transform the feature array into its principal component representation. The proposed solution requires to compute only three features for each spatial frequency.

We also considered the possibility of increasing or reducing the number $l$ of features in $\mathbf{f}$. Additional Figs $3(\mathrm{~b})$ and $3(\mathrm{c})$ report the average accuracy of the detector when $l=2\left(\mathbf{f}=\left[p_{F_{h}, m}\left(k_{1}\right) p_{F_{h}, m}\left(k_{2}\right)\right]_{F_{h}}\right)$ and $l=$ $4\left(\mathbf{f}=\left[p_{F_{h}, m}\left(k_{1}\right) p_{F_{h}, m}\left(k_{2}\right) p_{F_{h}, m}\left(k_{3}\right) p_{F_{h}, m}\left(k_{4}\right)\right]_{F_{h}}\right)$, respectively. Note that this would lead to feature arrays of 18 and 36 elements. The adopted set $k_{1}, \ldots, k_{l}$ of probability values is changed as for Fig. 3 (a) and each configuration is indexed by the values $\left(k_{1}-1\right)+\left(k_{2}-1\right) 9$ for $l=2$ and $\left(k_{1}-1\right)+\left(k_{2}-1\right) 9+\left(k_{3}-1\right) 81+\left(k_{4}-1\right) * 729$ for $l=4$. Indexes are reported on the $x$ axes.

It is evident that using 3 values proves to be the best solution since the maximum average accuracy is higher than using 2 or 4 probability values. In the first case, the feature space does not allow an effective clustering, whereas in the second the additional feature does not improve the classification accuracy.

As a results, each image is represented with a feature vector $\mathbf{f}$ of 27 elements (i.e., approximately six times smaller than in [18]).

The following section will show how the final classifiers are designed starting from the feature vectors $\mathbf{f}$. 


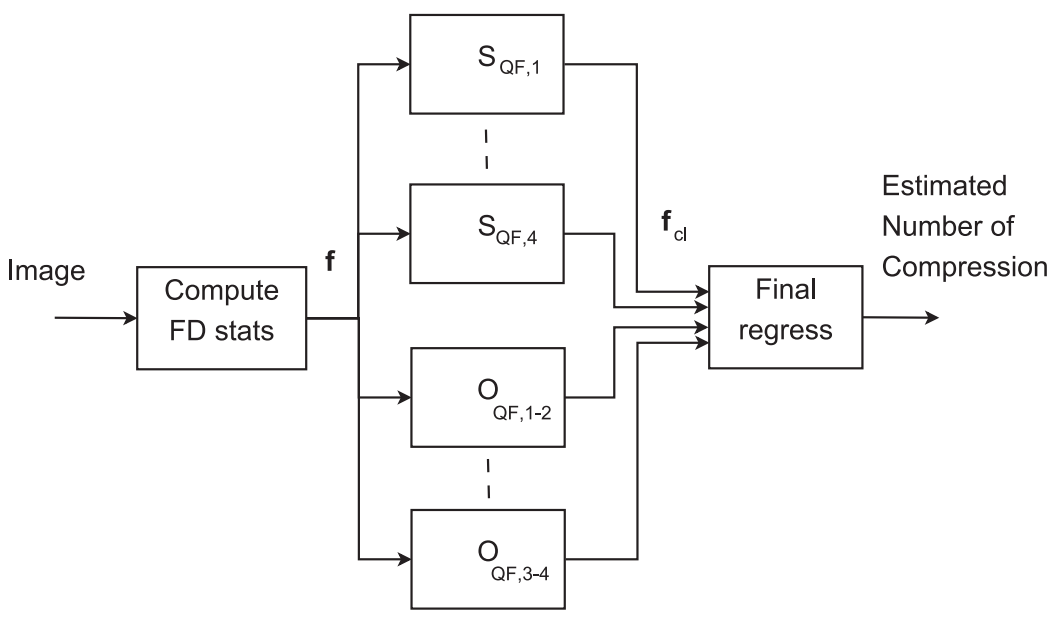

Fig. 4. Block diagram of the proposed detector.

\section{B) Design of the classifiers}

Given the dataset $D_{0}$, we computed the features $\mathbf{f}$ with $k_{1}=2, k_{2}=5$, and $k_{3}=6$ for every image. The dataset was divided into two subsets $D_{0}^{t}, D_{0}^{v}$ to train $\left(D_{0}^{t}\right)$ and validate $\left(D_{0}^{v}\right)$ the detector, respectively.

Assuming that the quality factor $Q F$ of the last compression stage is known (from the available bitstream), we built a set of $N_{T}$ binary SVM classifiers, $\mathcal{S}_{Q F, k}\left(k=1, \ldots, N_{T}\right)$, where each classifier $\mathcal{S}_{Q F, k}$ is able to detect whether the input image has been coded $k$-times or not.

In designing $\mathcal{S}_{Q F, k}$, we adopted the exponential kernel

$$
K\left(\mathbf{f}_{i}, \mathbf{f}_{j}\right)=\exp -\gamma_{k}\left(\left\|\mathbf{f}_{i}-\mathbf{f}_{j}\right\|_{2}^{\gamma_{k}}+1\right)
$$

The parameter $\gamma_{k}$ and the kernel type were optimized in the training phase computing the structure that gives the optimal performance. In this process, we adopted a crossvalidation optimization based on the RANSAC algorithm. The training is carried out looking for the best $\gamma_{k}$ value that maximizes the recall value for each class, i.e., maximizing the probability of correct classification for each subset of training images coded $N$ times. Each classifier also outputs a confidence value $w_{k}$ that reports the distance from the discriminating hyperplane and permits evaluating the reliability of the classification.

Similarly, second set of classifiers, named $O_{Q F, k-t}$, is generated which discriminate whether an images has been coded either $k$ or $t$ times. Naming $s_{k}$ the output of the classifier $S_{Q F, k}$ and $o_{k-t}$ the output of $O_{Q F, k-t}$, the outcomes of the different classifiers are combined together in order to make the estimation more robust.

Outputs are combined in the feature vector

$$
\mathbf{f}_{c l}=\left[s_{1} s_{2} s_{3} s_{4} o_{1-2} o_{1-3} o_{1-4} o_{2-3} o_{2-4} o_{3-4}\right]
$$

which has to be related to the number of compression. It is possible to relate $\mathbf{f}_{c l}$ to the unknown number of compressions $N$ that were applied to the analyzed image by training an regressor processing $\mathbf{f}_{c l}$. Figure 4 reports the whole detection scheme.
Performance was tested on the validation dataset $D_{0}^{v}$ and results are reported in the following section. A similar procedure was applied on the datasets $D_{1}$ and $D_{2}$.

\section{EXPERIMENTAL RESULTS}

As it was reported in the previous section, the detector was designed from a training database of images compressed up to four times. In order to generate the training database, we randomly selected the 100 images from the UCID dataset [25]. Each image was coded up to four times with a random sequence of quality factors $Q F_{i}$ for each compression stage. The quantization factor $Q F_{i}$ at stage $i$ is randomly chosen in the interval $\left[Q F_{i+1}^{l}-12, Q F_{i+1}^{l}-6\right] \cup\left[Q F_{i+1}^{u}+\right.$ 6, $\left.Q F_{i+1}^{u}+12\right]$, where $Q F_{i+1}^{l}$ and $Q F_{i+1}^{u}$ are the lower and upper $\mathrm{QF}$ limits that ensure that the quantization steps related to $Q F_{i}$ differ with respect to those of $Q F_{i+1}$.

These images were included in the datasets $D_{0}^{t}, D_{1}^{t}$, and $D_{2}^{t}$. A set of 200 images among the remaining pictures of the UCID dataset were compressed in the same ways and included in the datasets $D_{0}^{v}, D_{1}^{v}$, and $D_{2}^{v}$.

Since the dataset was extended with respect to the tests in [6], the performance proves to be slightly worse on average despite the approach proves to be effective in many cases. To provide evidence for this, we make available a demo version of the multiple compression detection algorithm for download at [26].

Table 1(a) shows a confusion matrix for dataset $D_{0}$. The real number of compression stages is reported along the rows while the estimated number of compressions is reported along the columns. Each cell $(u, v)$ reports the percentage of images compressed $u$ times that were detected as compressed $v$ times. It is possible to notice that the case of a single compression stage is always correctly identified. In the case an image was compressed 1, 2 or 3 times, the proposed method performs quite well since the probability of correct detection is above $84.8 \%$. The average accuracy is around $83 \%$. We compare the performance with that of a classifier designed adapting the work in [18] in order to detect the number of compression stages. The confusion 
Table 1. Confusion matrix for $Q F_{N}=75$ and dataset $D_{0}$. (a) Proposed method, (b) classifier in [18] (adapted).

\begin{tabular}{lrrrr}
\hline$N, N^{*}$ & 1 & 2 & \multicolumn{1}{c}{3} & \multicolumn{1}{c}{4} \\
\hline (a) & & & & \\
1 & $99.58 \%$ & $0.00 \%$ & $0.42 \%$ & $0.00 \%$ \\
2 & $10.00 \%$ & $84.46 \%$ & $5.54 \%$ & $0.00 \%$ \\
3 & $0.00 \%$ & $12.66 \%$ & $69.90 \%$ & $17.45 \%$ \\
4 & $0.00 \%$ & $10.02 \%$ & $20.13 \%$ & $79.85 \%$ \\
$(\mathrm{~b})$ & & & & \\
1 & $94.56 \%$ & $0.00 \%$ & $0.84 \%$ & $4.60 \%$ \\
2 & $0.56 \%$ & $13.06 \%$ & $81.13 \%$ & $5.25 \%$ \\
3 & $0.00 \%$ & $0.00 \%$ & $50.58 \%$ & $49.42 \%$ \\
4 & $0.00 \%$ & $0.00 \%$ & $12.46 \%$ & $87.54 \%$ \\
\hline
\end{tabular}

Table 2. Confusion matrix for $Q F_{N}=80$ and dataset $D_{0}$. (a) Proposed method, (b) classifier in [18] (adapted).

\begin{tabular}{lrrrr}
\hline$N, N^{*}$ & \multicolumn{1}{c}{1} & \multicolumn{1}{c}{3} & \multicolumn{1}{c}{4} \\
\hline (a) & & & & \\
1 & $100.00 \%$ & $0.00 \%$ & $0.00 \%$ & $0.00 \%$ \\
2 & $2.09 \%$ & $94.18 \%$ & $1.52 \%$ & $2.21 \%$ \\
3 & $0.20 \%$ & $1.52 \%$ & $71.23 \%$ & $27.05 \%$ \\
4 & $0.00 \%$ & $0.92 \%$ & $12.75 \%$ & $86.32 \%$ \\
$(\mathrm{~b})$ & & & & $3.20 \%$ \\
1 & $96.40 \%$ & $0.00 \%$ & $0.40 \%$ & $3.86 \%$ \\
2 & $0.23 \%$ & $93.40 \%$ & $2.51 \%$ & $32.69 \%$ \\
3 & $0.00 \%$ & $0.03 \%$ & $67.28 \%$ & $83.23 \%$ \\
4 & $0.00 \%$ & $0.00 \%$ & $16.77 \%$ & \\
\hline
\end{tabular}

matrix, which is shown in Table $1(b)$, demonstrates that the two methods achieve nearly the same results for $N=1$, but the average accuracy of the second solution is lower $(\sim 62 \%)$ with respect to the proposed solution. In this latter case, a significant performance loss is evident for $N=2$. This is due to the difficulty in identifying an adequate set of support vectors, because of the high dimensionality of feature vectors and the increased amount of compression noise introduced. The performance increases for $N>2$ since the availability of the full pmf of the FDs permits including those features that present wider oscillations whenever the image is compressed three or four times (e.g., $p(2)$ and $p(5)$ as Figs 2(c) and 2(d) show).

Results for quality factor $Q F=80$ are reported in Table 2. In this case, the average precision is $88 \%$ but the performance of the detector in identifying $N=3$ or 4 compressions improves. In this case, the adopted quantization steps are smaller and the amount of compression noise is lower permitting a more accurate detection even after more than two compression stages. Also in this case the approach in [18] (adapted to detect multiple compressions) presents a lower accuracy $(\sim 85 \%)$.

Table 3 reports the confusion matrix when $Q F_{N}=90$. It is possible to notice that the difference between the performances of the two approaches is significantly reduced. This fact is mainly correlated with the percentage of nonnull quantized coefficients in each image. At low QF values, many coefficients are quantized to zero, and therefore, the FD probabilities are computed from a reduced amount of
Table 3. Confusion matrix for $Q F_{N}=90$ and dataset $D_{0}$. (a) Proposed method, (b) classifier in [18] (adapted).

\begin{tabular}{lrrrr}
\hline$N, N^{*}$ & \multicolumn{1}{c}{1} & \multicolumn{1}{c}{3} & \multicolumn{1}{c}{4} \\
\hline (a) & & & & \\
1 & $100.00 \%$ & $0.00 \%$ & $0.00 \%$ & $0.00 \%$ \\
2 & $0.00 \%$ & $99.65 \%$ & $0.35 \%$ & $0.00 \%$ \\
3 & $0.00 \%$ & $0.46 \%$ & $99.19 \%$ & $0.35 \%$ \\
4 & $0.39 \%$ & $0.39 \%$ & $1.19 \%$ & $98.03 \%$ \\
(b) & & & & \\
1 & $100.00 \%$ & $0.00 \%$ & $0.00 \%$ & $0.00 \%$ \\
2 & $0.15 \%$ & $98.25 \%$ & $0.85 \%$ & $0.75 \%$ \\
3 & $0.00 \%$ & $0.38 \%$ & $99.48 \%$ & $0.15 \%$ \\
4 & $0.00 \%$ & $0.22 \%$ & $0.78 \%$ & $99.00 \%$ \\
\hline
\end{tabular}

Table 4. Confusion matrix with $Q F_{N}=80$ on dataset $D_{0}$ for the detection of five compression stages (proposed algorithm).

\begin{tabular}{lrrrrr}
\hline$N, N^{*}$ & 1 & \multicolumn{1}{c}{2} & 3 & 4 & \multicolumn{1}{c}{5} \\
\hline 1 & $100.00 \%$ & $0.00 \%$ & $0.00 \%$ & $0.00 \%$ & $0.00 \%$ \\
2 & $0.00 \%$ & $100.00 \%$ & $0.00 \%$ & $0.00 \%$ & $0.00 \%$ \\
3 & $0.00 \%$ & $5.33 \%$ & $70.67 \%$ & $22.67 \%$ & $1.33 \%$ \\
4 & $0.00 \%$ & $2.67 \%$ & $10.67 \%$ & $78.67 \%$ & $8.00 \%$ \\
5 & $0.00 \%$ & $0.00 \%$ & $36.00 \%$ & $10.67 \%$ & $53.33 \%$ \\
\hline
\end{tabular}

coefficients. Since in this case the statistics is diluted, many probability values with $l=8$ are not reliable features for the classification. Shrinking the array $\mathbf{f}$ to the subset of the most reliable features permits increasing the accuracy of the detection.

In the end, we also evaluated the possibility of detecting five compression steps for the images adopted to generate dataset $D_{0}$. In this case, confusion matrix for the proposed approach is reported in Table 4. It is possible to notice that, although the average accuracy is decreased to $80 \%$, the capability of detecting $N<5$ compression stages is increased. This fact is mainly due to the fact that the detectors are trained with images coded more than four times, and as a consequence, the partitionings of features space operated by the SVM classifiers are more accurate.

It is possible to notice that the accuracy of the method based on [18] (adapted) increases as the compression quality increases. This fact is mainly correlated with the percentage of non-null quantized coefficients in each image.

At low $Q F$ values, many coefficients are quantized to zero, and therefore, many probability values $\hat{p}_{F_{h}, m}(k)$ are computed from a reduced set of data and do not provide reliable features for the classification. Shrinking the array $\mathbf{f}$ to the subset of the most reliable features permits increasing the accuracy of the detection. It is possible to notice that at high QF values (low distortion) the accuracy of the approach in [18] get closer to that of the proposed approach.

The proposed method is characterized by a lower computational complexity with respect to the approach in [18], since the size of the feature vector is much smaller.

We also tested the improved accuracy of the proposed classifier in a simple double compression detection (i.e., verifying whether an image has been compressed once or more 

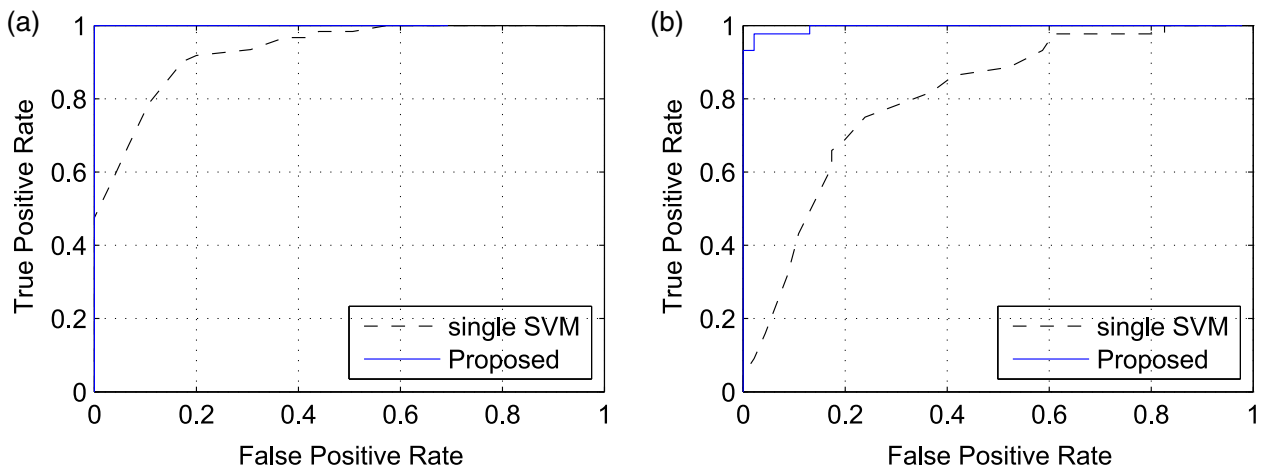

Fig. 5. ROC curves of different double compression detectors for different QFs on dataset $D_{0}$. (a) QF $=70$, (b) QF $=80$.

Table 5. Computational time for the proposed method and the classifier in [18] (adapted).

\begin{tabular}{lccc}
\hline $\begin{array}{l}\text { Computational } \\
\text { time }\end{array}$ & $\begin{array}{r}\text { Proposed } \\
(\mathrm{ms})\end{array}$ & $\begin{array}{r}\text { Li } \text { et al. } \\
(\mathrm{ms})\end{array}$ & $\begin{array}{r}\text { Saving } \\
(\%)\end{array}$ \\
\hline $\mathrm{QF}=70$ & 0.55 & 1.19 & 53.78 \\
$\mathrm{QF}=80$ & 1.13 & 2.18 & 48.16 \\
\hline
\end{tabular}

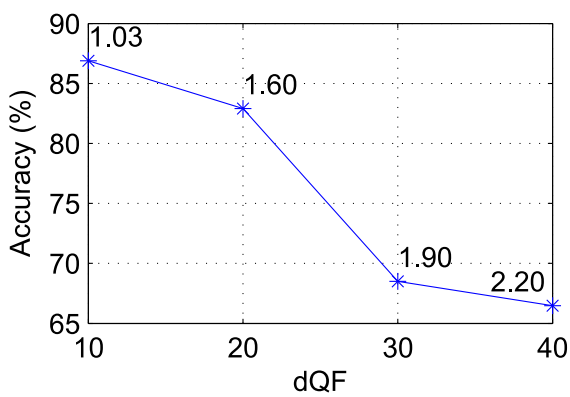

Fig. 6. Accuracy versus $d Q F$ for images coded with $Q F_{N}=70$ on dataset $D_{0}$. The graph also reports the average PSNR decrement $(\mathrm{dB})$ for each point.

Table 6. Confusion matrix for $Q F_{N}=80$ from datasets $D_{1}$. (a) Proposed method, (b) classifier in [18] (adapted).

\begin{tabular}{lrrrr}
\hline$N, N^{*}$ & 1 & 2 & 3 & \multicolumn{1}{c}{4} \\
\hline (a) & & & & \\
1 & $98.84 \%$ & $1.16 \%$ & $0.00 \%$ & $0.00 \%$ \\
2 & $0.00 \%$ & $71.45 \%$ & $20.41 \%$ & $8.14 \%$ \\
3 & $0.00 \%$ & $19.51 \%$ & $52.33 \%$ & $28.17 \%$ \\
4 & $0.00 \%$ & $20.03 \%$ & $40.56 \%$ & $39.41 \%$ \\
(b) & & & & \\
1 & $100.00 \%$ & $0.00 \%$ & $8.14 \%$ & $0.00 \%$ \\
2 & $0.00 \%$ & $74.21 \%$ & $15.48 \%$ & $10.32 \%$ \\
3 & $0.00 \%$ & $16.15 \%$ & $57.24 \%$ & $26.61 \%$ \\
4 & $0.00 \%$ & $16.28 \%$ & $45.87 \%$ & $37.86 \%$ \\
\hline
\end{tabular}

times). More precisely, tested the SVM classifier proposed in [18] with respect to the classifier using the features in equation (8), which compose different SVM classifiers using a reduced set of features. Figure 5 reports the receiver operating characteristic (ROC) curves of the two approaches for different QFs. It is possible to see that the proposed detector is much more effective in detecting double compression.
Table 7. Confusion matrix for $Q F_{N}=80$ from datasets $D_{2}$. (a) Proposed method, (b) classifier in [18] (adapted).

\begin{tabular}{lcrrc}
\hline$N, N^{*}$ & 1 & \multicolumn{1}{c}{2} & \multicolumn{1}{c}{3} & \multicolumn{1}{c}{4} \\
\hline (a) & & & & \\
1 & $98.84 \%$ & $1.16 \%$ & $0.00 \%$ & $0.00 \%$ \\
2 & $0.00 \%$ & $92.49 \%$ & $7.51 \%$ & $0.00 \%$ \\
3 & $0.00 \%$ & $20.72 \%$ & $55.92 \%$ & $23.36 \%$ \\
4 & $0.00 \%$ & $22.30 \%$ & $33.30 \%$ & $44.40 \%$ \\
(b) & & & & \\
1 & $91.86 \%$ & $0.00 \%$ & $8.14 \%$ & $0.00 \%$ \\
2 & $0.00 \%$ & $84.14 \%$ & $15.86 \%$ & $0.00 \%$ \\
3 & $0.00 \%$ & $17.65 \%$ & $43.34 \%$ & $39.01 \%$ \\
4 & $0.00 \%$ & $17.55 \%$ & $23.57 \%$ & $58.88 \%$ \\
\hline
\end{tabular}

Furthermore, we considered the robustness of the approach changing the range of variation for the QF parameters. More precisely, we changed the width $d Q F$ of the interval where QF values are randomly chosen. Figure 6 reports the average accuracy obtained changing the width of the interval as a function of $d Q F$. It is possible to notice that increasing the range of $\mathrm{QF}$ values leads to a decrement of the detection performance and of the average peak signal-to-noise ratio (PSNR) value after $N$ coding stages.

Finally, we also tested the robustness of the approach in case an image is manipulated between consecutive compression stages. More precisely, we assumed that image manipulation took place at the processing block in Fig. 1, before one of the coding stages.

In the processing chain, we introduced a random manipulation which could consist either in a rescaling or in a rotation. The idea is to disalign the block grid of JPEG compression in order to test the robustness of the detector. The parameter values for each transformation are random as well and can vary in the intervals $[.9,1.1]$ for the rescaling (the parameter is the pixel width ratio), and $[-5,5]$ for the rotation angle. These limits are justified by the need of avoiding a significant decrement of the image quality and keeping the size of the transformed image close to that of the original one. These data were collected in the datasets $D_{1}$ and $D_{2}$.

The confusion matrix in Table 6 was obtained from dataset $D_{1}$ and show that the proposed classifier permits obtaining an average accuracy of $65 \%$. Note also that 
increasing the size of the feature vector $\mathbf{f}$ (see Table 6(b)) does not bring significant improvements in terms of performance, made exception for the detection of three compression stages. The approach in [18] permits obtaining an average accuracy equal to $62 \%$ in presence of random rescalings.

As for the dataset $D_{2}$, Table 7 shows that the proposed solution permits obtaining an average accuracy equal to $75 \%$, while the solution derived from [18] permits having an average accuracy of $69 \%$.

\section{CONCLUSIONS}

The paper describes a classification strategy that permits detecting the number of JPEG compression stages performed on a single image. The approach relies on a set of SVM classifiers applied to features based on the statistics of the FDs of quantized DCT coefficients. The proposed solution performs well with respect to previous approaches, while employing a reduced set of features. Future research will be devoted to investigate other possible antiforensics strategies that could fool the proposed solution and to extend the approach to the case of video signals.

\section{ACKNOWLEDGEMENTS}

The project REWIND acknowledges the financial support of the Future and Emerging Technologies (FET) programme within the Seventh Framework Programme for Research of the European Commission, under FET-Open grant number, 268478 .

\section{REFERENCES}

[1] Piva, A.: An overview on image forensics. ISRN Signal Processing, 2013, 2013, article ID 496701.

[2] Milani, S. et al.: An overview on video forensics. APSIPA Trans. Signal Inf. Process., 1 (12) (2012).

[3] Farid, H.: Exposing digital forgeries in scientific images, in Proc. of the 8th Workshop on Multimedia and Security (MMeSec 2006), September 26-27, 2006, pp. 29-36.

[4] Lukás, J., Fridrich, J.: Estimation of primary quantization matrix in double compressed JPEG images, in Proc. DFRWS, Cleveland, OH, USA, August 2003.

[5] Wallace, G.: The JPEG Still Picture Compression Standard, Commun. ACM, 34 (4) (1991), 30-44.

[6] Milani, S.; Tagliasacchi, M.; Tubaro, M.: Discriminating multiple JPEG compression using first digit features, in Proc. 37th Int. Conf. on Acoustics, Speech, and Signal Processing (ICASSP 2012), Kyoto, Japan, March 25-30, 2012, pp. 2253-2256.

[7] Pevny, T.; Fridrich, J.: Estimation of primary quantization matrix for steganalysis of double-compressed JPEG images, in Proc. SPIE, 6819, San Jose, CA, USA, January 2008, pp. 11-1-11-13.

[8] Galvan, F.; Puglisi, G.; Bruna, A.R.; Battiato, S.: First quantization matrix estimation from double compressed JPEG images. IEEE Trans. Inf. Forensics Security, 9 (8) (2014) 1299-1310.
[9] Benford, F.: The law of anomalous numbers. Proc. Am. Philos. Soc., 78 (4) (1938) 551-572, jSTOR 984802.

[10] Pérez-González, F., Heileman, G.L.; Abdallah, C.T.: Benford's law in image processing, in Proc. of IEEE Int. Conf. Image Processing (ICIP 2007), San Antonio, TX, USA, September 2007.

[11] Lam, E.Y.; Goodman, J.W.: A mathematical analysis of the DCT coefficient distributions for images. IEEE Trans. Image Process., 9 (10) (2000) 1661-1666.

[12] Wang, W.; Farid, H.: Exposing digital forgeries in video by detecting double JPEG compression, in Proc. of ACM MMぬSec 2006, Geneva, Switzerland, September 2006, pp. 37-47.

[13] Lin, Z.; He, J.; Tang, X.; Tang, C.-K.: Fast, automatic and fine-grained tampered JPEG image detection via DCT coefficient analysis. Pattern Recognit., 42 (11) (2009) 2492-2501.

[14] Fu, D.; Shi, Y.Q.; Su, W.: A generalized Benford's law for JPEG coefficients and its applications in image forensics, in Proc. of SPIE, 6505, January 28-Februbay 1, 2009, pp. 39-48.

[15] Xian-Zhe, M.N. Shao-Zhang, Jian-Chen, Z.: Tamper detection for shifted double JPEG compression, in Proc. IIH-MSP 2010, Darmstadt, Germany, October 2010, pp. 434-437.

[16] Barni, M.; Costanzo, A.; Sabatini, L.: Identification of cut \& paste tampering by means of double-JPEG detection and image segmentation, in Proc. of ISCAS 2010, Paris, France, May 30-June 2, 2010, pp. 1687-169o.

[17] Qu, Z.; Luo, W.; Huang, J.: A convolutive mixing model for shifted double JPEG compression with application to passive image authentication,' in ICASSP. IEEE, 2008, pp. 1661-1664. [Online]. Available: http://dx.doi.org/10.1109/ICASSP.2008.4517946

[18] Li, B.; Shi, Y.Q.; Huang, J.: Detecting doubly compressed JPEG images by using mode based first digit features', in Proc. of MMSP 2008, Cairns, Queensland, Australia, October 2008, pp. $730-735$.

[19] Bianchi, T.; Rosa, A.D.; Piva, A.: Improved dct coefficient analysis for forgery localization in JPEG images, in Proc. of the 36th Int. Conf. on Acoustics, Speech, and Signal Processing (ICASSP 2011), Prague, Czech Republic, March 22-27, 2011, pp. 2444-2447.

[20] Wang, J.; Cha, B.; Cho, S.; Kuo, C.: Understanding Benford's law and its vulnerability in image forensics, in Proc. of the 2009 IEEE Int. Conf. on Multimedia and Expo (ICME 2009), June 28-July 1, 2009, pp. 1568-1571.

[21] Battersby, S.: Statistics hint at fraud in Iranian election. New Scientist, no. 2714, June 2009. [Online]. Available: http://www. newscientist.com/article/mg20227144.0oo-statistics-hint-at-fraud-iniranian-election.html

[22] Deckert, J.; Myagkov, M.; Ordeshook, P.C.: The irrelevance of Benford's law for detecting fraud in elections. Caltech/MIT Voting Technology Project, no. Working paper no. 9, September 2010. [Online]. Available: http://www.vote.caltech.edu/drupal/node/327

[23] Nigrini, M.J.: I’ve got your number. J. Account., May 1999. [Online]. Available: http://www.journalofaccountancy.com/Issues/1999/May/ nigrini

[24] Müller, H.C.: Greece was lying about its budget numbers. Forbes, September 2011. [Online]. Available: http://www.forbes.com/ sites/timworstall/2011/09/12/greece-was-lying-about-its-budgetnumbers/

[25] Schaefer, G.; Stich, M.: UCID - An uncompressed colour image database, in Proc. SPIE, vol. 5307, San Jose, CA, USA, January 2004, pp. $472-480$.

[26] Milani, S.: Multiple JPEG Compression Detector Website, December 2014. [Online]. Available: http://www.dei.unipd.it/simımil/materiale/ mjpegdetector/ 
Simone Milani is currently Assistant Professor at the University of Padova, Italy. From this institution, he received the Laurea degree in Telecommunication Engineering in 2002, and the Ph.D. degree in Electronics and Telecommunication Engineering in 2007. In 2006, he was a visiting Ph.D. student at the University of California, Berkeley under the supervision of Professor K. Ramchandran. In 2007, he was a Post-doc researcher at the University of Udine, Italy, collaborating with Professor R. Rinaldo. In 2011, he joined the group of Professor Stefano Tubaro at the Politecnico di Milano, Italy working as an assistant researcher within the ICT-FP7 FET-Open Project REWIND. Dr. Milani co-authored more than 70 papers in international journals and conferences, including award winning papers at MMSP 2013, MMSP2012. His main research topics are digital signal processing, image, and video coding, $3 \mathrm{D}$ image and video acquisition and processing, $3 \mathrm{DTV}$ compression, multimedia forensics.

Marco Tagliasacchi is currently an Assistant Professor at the "Dipartimento di Elettronica e Informazione - Politecnico di Milano", Italy. He received the "Laurea" degree (2002, cum Laude) in Computer Engineering and the Ph.D. in Electrical Engineering and Computer Science (2006), both from Politecnico di Milano. He was visiting academic at the Imperial College London (2012) and visiting scholar at the University of California, Berkeley (2004). His research interests include multimedia forensics, multimedia communications (visual sensor networks, coding, quality assessment), and information retrieval. Dr. Tagliasacchi co-authored more than 120 papers in international journals and conferences, including award winning papers at MMSP 2013, MMSP2012, ICIP 2011, MMSP 2009, and QoMex 2009. He has been actively involved in several EU-funded research projects. $\mathrm{He}$ is currently co-coordinating two ICT-FP7 FET-Open projects (GreenEyes - www.greeneyesproject.eu, REWIND www.rewindproject.eu).

Stefano Tubaro completed his studies in Electronic Engineering at the Politecnico di Milano, Italy, in 1982. Since December 2004 he has been appointed as Full Professor of Telecommunication at the Dipartimento di Elettronica, Informazione e Bioingegneria of the Politecnico di Milano (DEIB-PoliMi). His current research interests are on advanced algorithms for video and sound processing. Stefano Tubaro authored over 150 publications on international journals and congresses. In the past few years, he has focused his interest on the development of innovative techniques for image and video tampering detection and, in general, for the blind recovery of the "processing history"I of multimedia objects. Stefano Tubaro is the Head of the Telecommunication Section of DEIB-PoliMi, and the Chair of the IEEE SPS Italian Chapter; moreover he coordinates the research activities of the Image and Sound Processing Group (ISPG). He is a member of the IEEE MMSP and IVMSP TCs. 